\title{
DEVELOPMENT OF A TACTICAL GUIDANCE RESEARCH AND EVALUATION SYSTEM (TGRES)
}

\author{
Kenneth H. Goodrich ${ }^{*}$, John W. McManus ${ }^{+}$ \\ NASA Langley Research Center, Hampton, Virginia
}

\begin{abstract}
The development of a simulation tool for tactical guidance research and the evaluation of systems performance in a tactically significant environment is described. The objective of the tool is to provide a means by which researchers can explore and exploit enhancements to airplane agility. The completed tool will include high-fidelity batch and piloted simulation capabilities, an advanced tactical guidance logic and a user-friendly interface. While the tool is being developed for the purpose of studying airplane agility, its modularity should make it easily adaptable to the analysis of other technologies and thus be of interest to a number of potential users.
\end{abstract}

\section{Nomenclature}

$\begin{array}{ll}\text { AML } & \text { - Adaptive Maneuvering Logic } \\ \text { COSMIC } & \text { - Computer Software Management and Information Center } \\ \text { DMS } & \text { - Differential Maneuvering Simulator } \\ \text { SA } & \text { - superagility } \\ \text { TDG } & \text { - Tactical Decision Generator } \\ \text { TGRES } & \text { - Tactical Guidance Research and } \\ & \quad \text { Evaluation System } \\ \text { TMS } & \text { - Tactical Maneuvering Simulator } \\ \text { wvr } & \text { - within visual range } \\ 6 \text { dof } & \text { - six degrees of freedom }\end{array}$

\section{Introduction}

As new technologies or capabilities are proposed for inclusion in high performance aircraft, it is imperative to assess the utilization and impact of these additions within the context of air to air 
combat. This assessment can be a difficult task, particularly when faced with technologies which are well outside the range of current experience. A case in point is superagility (SA). SA is defined as enhanced maneuverability and controllability throughout an expanded flight envelope and is usually achieved through the blending of conventional and advanced control effectors (e.g. thrust vectoring, forebody strakes) with a highly integrated control system. SA facilitates unconventional maneuver options like post-stall maneuvering (i.e. supermaneuverability), roll about the velocity vector under load, and decoupled flight. Of course, this new capability is not without drawbacks as well. Designs will be more complex with potential increases in size, weight, and cost, and decreases in other measures of performance. In addition, the increased physical and mental stresses placed on the pilot by SA may limit the realizable performance of the man/machine system.

Herbst $^{1}$, Foltyn ${ }^{2}$, Pennington ${ }^{3}$ and others have tried to demonstrate the utility of various aspects of SA through theoretical analysis and simulation. While this work has done a great deal to reveal the potential of SA, controversy still remains about its use and impact in tactically meaningful scenarios. To date, many of these studies have been restricted to simplified airplane models (e.g. point mass). While perfectly valid results can (and should) be obtained from such analysis, in an area such as SA where the nonlinear effects of large amplitude maneuvers often have first order effects, these results need to be validated through more sophisticated analysis. Currently this process is frequently left undone do to lack of the proper simulation tool. Also, most studies have been limited to a single airplane or a duel between opposing aircraft because an applicable means of simulating and guiding multiple aircraft during within visual range (wvr) combat has not existed. This limitation has left unanswered concerns that the tactics and benefits of SA seen in 1 versus 1 engagements will not carry-over into engagements with more than two aircraft. Similarly, lack of suitable analysis tools has restricted the study of trade-offs between SA and other, potentially conflicting characteristics such as availability and observability, thus preventing SA from being evaluated in a comprehensive manner.

To help address these issues NASA Langley Research Center is developing the Tactical Guidance Research and Evaluation System (TGRES, pronounced 'tigress'). TGRES is similar in intent to existing digital air combat simulations such as the Adaptive Maneuvering Logic (AML) TAC Brawler ${ }^{5}$, or AASPEM, enabling the study of airplane combat tactics or systems in a realistic, but controlled and repeatable environment. These systems allow the researcher to define the initial conditions of an aircombat engagement by specifying airplane models, tactical doctrines, guidance logics, and initial airplane states. Using a batch simulation process, with the possible potential for occasional operator intervention, the engagement evolves under the specified rules and constraints. By performing a parametric analysis over one of the input quantities, the effect of this quantity can be evaluated. TGRES differs from existing systems by capitalizing on developments in computing techniques to increase system capability and flexibility, and by incorporating six 
degrees of freedom (6 dof) airplane dynamics. A further feature of the system is the inclusion of Langley Research Center's Differential Maneuvering Simulator, providing the ability to include experienced pilots in real-time evaluations.

This paper will describe the development and current status of TGRES.

\section{Overview of TGRES}

TGRES consists of three main elements, the Tactical Decision Generator (TDG), the Tactical Maneuver Simulator (TMS) and the Differential Maneuvering Simulator (DMS) as shown in figure 1. The TMS and DMS provide simulation environments, while the TDG is a knowledge-based guidance system exercised within these environments. The intended use of the system is to systematically determine the benefits of SA and to develop tactics which exploit these benefits. This is being accomplished by first developing and analyzing tactics in a batch simulation mode using the TDG and the TMS.

Each time a change or enhancement is made to the TDG, a series of 64 engagements with varying initial conditions are simulated in batch using the TMS. The effects of the change are evaluated by studying the resulting trajectories and statistics compiled during each engagement. These statistics include first shot opportunities, time on offense with advantage, probability of survival and accumulated time with the opponent in the gun and missile envelopes. After the effects of the change are understood, further modifications are made and evaluated in an effort to maximize the benefits of a given change. After the logic has converged to a stable configuration, a more comprehensive set of 320 initial conditions are run and the results are evaluated. If the results of these batch simulations show the expected level of improvement, the modified guidance logic is then evaluated in manned simulation in the DMS.

A series of engineering and test pilots are used as opponents to fly against the TDG in real time. Each pilot completes a standardized sequence of initial engagement conditions including advantaged, disadvantaged and neutral positions. The results of these engagements are evaluated using the same measures of performance used in the batch analysis, with the addition of pilot comments and suggestions. Candidate modifications arising from the manned simulations are incorporated into the TDG and the batch and manned evaluation processes are repeated. The net result of this research will be a heightened understanding of the fundamental characteristics of SA and how these characteristics can be exploited to achieve maximum effectiveness.

The modularity TGRES is such that the elements of the system can be implemented in computationally favorable environments. The TDG is being developed and implemented on a Symbolics 3650 using Lisp as the programming language. The TMS is being developed and implemented on a VAX 3200 workstation using conventional programming languages. The DMS 
currently uses a Cyber 175 mainframe computer and FORTRAN as the primary programming language. The elements execute as co-tasks and communicate via network connections. In addition to enhancing development and implementation efficiency, the task tailored computational environments provide a degree of parallel processing which reduces execution time.

The following three sections describe the individual elements of TGRES.

\section{Tactical Decision Generator}

The TDG is a knowledge-based guidance system programmed in Lisp and designed to explore the maneuvering options offered by SA. It currently utilizes the trial maneuver concept developed for the $\mathrm{AML}^{4}$ with several extensions. The trial maneuver concept involves predicting for a given decision interval, the future position, attitude and velocity of the opponent by extrapolating along a curve fit to the opponent's past positions. A series of 'elemental maneuvers' are then setup to determine potential future locations of the guided airplane. Currently, these elemental maneuvers consist of combinations of load factor, bank angle and thrust. A set of metrics defining a situation space is used to evaluate the worthiness of the elemental maneuvers and the most promising maneuver is then executed. The trial maneuver process, shown schematically in figure 2 , enables the TDG to construct complex trajectories similar to those produced by human pilots.

The TDG differs from the AML (as obtained from the Computer Software Management and Information Center - COSMIC) in having a more sophisticated evaluation logic. Where as the AML has a fixed set of 15 binary 'yes/no' tactical evaluation metrics or questions defining the situation space, the TDG uses 'fuzzy logic' to more accurately define the space. For instance, to the question, 'Is the opponent within my weapons envelope?' AML is restricted to either a yes or no ( 1 or 0 respectively) response. The TDG assigns a value between zero and one depending on the degree to which the opponent is in the envelope. A near zero value is assigned if the opponent is on the edge of the envelope and is increased until reaching a value of one as the opponent occupies the heart of the weapons envelope and the probability of kill is high. The TDG also uses distinct modes of operation to vary the relative importance of the individual metrics in response to a change in situation. The TDG contains a situation assessment module which evaluates the state of its own airplane, its current mission or objective, the relative geometry of the opponent, and the opponents instantaneous intent to determine the mode of operation. Each mode of operation has a unique set of weights which are applied to the individual scoring metrics, adjusting their relative importance. The modes of operation currently supported by the TDG are:

Aggressive

Defensive 
Neutral

Bugout

Evasive

ground/stall evasion

missile evasion

evade weapon lock of opponent

In addition to a unique set of scoring weights, each mode has a task tailored decision interval length. For example, while in the aggressive mode, the TDG is frequently fine tracking its opponent. During this tracking it has been found that a relatively short decision interval time $(\sim 0.5$ sec.) results in a more robust solution. During more neutral maneuvering, this same short decision interval time results in the TDG performing a 'thrashing' motion, unnecessarily bleeding energy and lowering effectiveness. Thus, during this mode, a longer interval ( $1.0 \mathrm{sec})$ is employed.

The TDG is currently being exercised using the airplane dynamics contained in the AML in both batch simulation and real-time simulation against pilots. The airplane model utilized from AML is a performance model, meaning that the thrust, lift, drag and turning capabilities are represented by their maximum, steady state values. Thus, the transient behavior of the airplane is not accurately modeled. During these exercises, data representing the characteristics of a modern high-performance fighter was used in the airplane models. Figure 3 (ref. 6) summaries the results of a series of engagements between the TDG and the decision logic contained in the Cosmic version of AML. Results of the real-time simulations are presented in the section on the DMS.

As the capability to simulate engagements with three or more aircraft, some capable of performing superagile maneuvers, becomes available through the TMS, the focus of the TDG development effort will center on adapting the system to perform in this more complex environment. Enhancements to the logic must be made to coordinate the efforts of cooperating aircraft as well as considering the threats presented by multiple hostile aircraft. Further more, the tactical evaluation metrics will need to be further refined to reflect the impact of SA. The current evaluation metrics tend to focus on locally optimum maneuvers; for example -- what should be done in the current decision interval to bring the opponent into the weapons envelope. SA will increase the number of maneuver options and while many of these new options may appear to be of immediate benefit, the long term consequences of these maneuvers (such as extremely high energy loss) may make them ineffective. The TDG needs to recognize and avoid these dead-end maneuvers.

More detailed information on the TDG can be found in reference 6.

Tactical Maneuver Simulator 
When Completed, The TMS will provide a unique, high-fidelity, batch aircombat simulation environment in which to develop and test various guidance strategies. The TMS will allow researchers to define the initial conditions between multiple aircraft and then control the trajectories and attitudes of the aircraft using simple trajectory commands or through a guidance system such as the TDG. The TMS consists of 3 sub-elements: a high-fidelity simulation, a tactical autopilot and a user interface and color graphics system.

The simulation sub-element provides for the simulation of the engagement participants and any missiles which may be in-flight. Airplane dynamics are being modeled using the simulation described in reference 7. This simulation uses the Advanced Continuous Simulation Language ${ }^{8}$ as the model building framework. Included in this package are the rigid-body characteristics of an advanced fighter aircraft with the option to include a two vane thrust-vectoring system. The user of the TMS will be able to specify the number, type (thrust-vectoring or non thrust-vectoring) and initial states of the engagement participants. While the TMS is being structured to allow any number of participants during a batch simulation, more than three aircraft may make the system unacceptably slow while executing on a single VAX workstation. A more powerful machine or parallel processing could be used to elevate this slowdown.

Unlike most existing batch, aircombat simulations, the airplane dynamics in the TMS are being modeled using a full 6 dof. The large amplitude maneuvers typical of aircombat maneuvering combined with the unusual attitudes enabled by superagility, make the use of 6 dof almost mandatory to accurately model the transient motions of the airplane. However, the use of 6 dof will place additional, complicating demands on the guidance element (in this case, the TDG) unless an interface is added to deal with these new degrees of freedom. In the TMS, the tactical autopilot provides this interface. The intent of the autopilot is to allow the guidance logic to operate with the minimum number of degrees of freedom required to specify the desired airplane trajectory, thus freeing the guidance logic from the burden of flying the 6 dof airplane simulation. The function of the tactical autopilot might be compared to the 'piloting' actions of a fighterpilot in the sense that it translates the trajectory and attitude commands issued by the guidance logic into control system inputs which cause the airplane to follow the desired path. The placement of the autopilot in TDG/airplane system is shown in figure 4. The location of the autopilot is intended to minimize the modifications needed to implement different control systems. It should be possible to substitute candidate control systems of the same family (i.e. rate command, attitude command etc...) into the simulation without modifying the autopilot, thus providing a means of comparing in batch simulation the impact of the control system on the tactical effectiveness of the airplane. For most conventional aircombat maneuvering, coordinated flight (i.e. minimum sideslip) is desired. Trajectories of this nature can be described by the guidance logic using bank angle, load factor, and thrust as outputs. An approach similar to that described in reference 9 is being used to design the 
tactical autopilot using these quantities as inputs. For unconventional maneuvering, it is frequently desired to command the attitude of the airplane. For instance, it may be possible to achieve a weapons lock by slewing the nose of the airplane to point directly at an opponent. A second mode which can be selected by the guidance logic is also being designed into the autopilot to support this type of maneuvering.

The user interface under development permits the TGRES user to examine the results of previously simulated engagements and to interact with engagements as they occur using a color graphics system with mouse and keyboard inputs. The engagement replay system shown in figure 5 allows the user to replay data recorded during engagements simulated using the TMS and DMS. The replay system plots the trajectories of the engagement participants on a three dimensional axis system which is surrounded by windows displaying the instantaneous and maximum values of key aircraft quantities such as altitude, Mach number and deviation angle from the opponent. In addition to the displays of the replay system, the interactive system enables the user to change the status of the airplanes systems using a mouse sensitive display. This capability allows the user to study the response of the guidance logic to the change in system status. Future enhancements to the user interface include the ability for the operator to manually input guidance commands directly for one or more of the airplanes, bypassing or overriding the guidance logic. By allowing this manual control, the TMS becomes a non-real time, man in the loop tactical workstation. Experienced pilots and tacticians could work with TGRES using this capability to interactively evaluate and improve the guidance logic. Engagements could be allowed to run under the control of the guidance logic, but under the eye of an experienced tactician. If the logic preformed a maneuver that did not seem sound, the operator could go back to the start of the maneuver and manually fly the airplane through the sequence, trying different options. After determining an effective maneuver or counter, the operator could then modify the guidance logic to reflect the experience gained during the engagement.

\section{Differential Maneuvering Simulator}

The third element of TGRES is the DMS. The DMS is a twin dome (40' in diameter) simulator located at Langley and is intended for real-time simulation of engagements between piloted aircraft. By using the TDG to drive one of the domes, the decision logic can be evaluated against an unpredictable and highly adaptive human opponent in real-time. This capability has proven invaluable during the development of the TDG. As was described in the section on the TDG, the development of the decision logic is done primarily in batch using the TMS. While the repeatability of the batch simulation provides an excellent environment for developing the decision logic, this same repeatability can cause difficulties. During the batch development process a 
baseline decision logic is generally used as the opponent. This opponent performs tactically sound maneuvers and within the constraints of its programming adapts to the maneuvers of the attacker. However, as the decision logic is restrained to proven tactics, it is possible for the logic to overlook an improbable, but effective maneuver. By using a number of human pilots as opponents in the DMS, these 'holes' are uncovered and eliminated by modifying the TDG. When the TMS is completed, much of this work can be done using the tactical workstation feature, but even with this capability, due to the natural interface the DMS affords to experienced pilots, the real-time simulator will remain a vital part of TGRES.

The performance of the TDG against an experienced engineering pilot is shown in figure 6 . The pilot used in the collection of this data has the highest experience level flying against the TDG and generally performs better than the average test subject. The engagements were started at the edge of the wvr envelope with the pilot being able to see the position of the TDG controlled airplane but often not being able to determine the attitude of the airplane until observing its motions after the start of the simulation. The initial conditions were presented in a random order to prevent the pilot from unfairly anticipating the initial actions of the automated opponent. The initial conditions are biased such that the pilot has a overall position advantage equivalent to an exchange ratio of about 1.4 , thus the

Future plans for the DMS include the addition of a third dome (20' diameter), enabling the simulation of engagements composed of up to three pilots or airplanes controlled by guidance logics. This addition will allow the TDG to be evaluated in real-time, one versus two scenarios, further enhancing the tactical realism and utility of TGRES.

\section{Concluding Remarks}

The completed TGRES will provide an integrated environment for developing tactical guidance algorithms and evaluating the impact of new technologies. While being developed to study SA, the modularity of the system will make it possible to easily modify or replace an element of the system in order to appraise other technologies. TGRES differs from existing systems by extensively capitalizing on Artificial Intelligence programming techniques in the implementation of the guidance logic. The TDG, the guidance element of TGRES, has been shown to perform significantly better than the COSMIC version of AML in wvr engagements. The system will also include a high-fidelity, batch simulation module, capable of accurately modeling airplane dynamics, including control system effects, throughout the flight envelope. It will be possible to asses the impact of these dynamics in tactically significant batch and real-time simulations, enabling optimization of the dynamics during the design process. In addition, the tactical workstation feature and the DMS facilitates natural interaction with tacticians and pilots, allowing the decision 
logic to be tested in an unpredictable environment. This capability has resulted in significant improvements to the decision logic by exposing deficiencies that would have been difficult to detect using purely batch simulations.

\section{References}

1. Herbst, W. B.: Supermaneuverability. MBB/FE1/S/PUB/120, 1983

2. Foltyn, R. W., et al.: Development of Innovative Air Combat Measures of Merit for Supermaneuverable Fighters. Eidetics Int., TR 87-211, 1987

3. Pennington, J. E. and Meintel, A. J., jr: Simulation Study of Thrust Vectoring for Air Combat Maneuvering for a Lightweight Fighter-Class Aircraft. NASA TM X-3270, 1975

4. Burgin, G. H., et al.: An Adaptive Maneuvering Logic Computer Program for the Simulation of One-on-One Air-to-Air Combat. Vol I and Vol II. NASA CR-2582, CR2583,1975

5. Kerchner, R. M., et al: The TAC BRAWLER Air Combat Simulation Analyst Manual (Rev. 3.0). Decision Science Applications Report \#668, 1985

6. McManus, J. W., and Goodrich, K. H.: Application of Artificial Intelligence (AI) Programming Techniques To Tactical Guidance for Fighter Aircraft. AIAA Paper 893525, August, 1989

7. Butrill, C.S., et al.:

8. Advance Continuous Simulation Language (ACSL) Reference Manual, Fourth Edition. Mitchell and Gauthier Associates, Condord, MA, 1986

9. Burgin, B. H., and Eggleston, D. M.: Design of an All-Attitude Flight Control System To Execute Commanded Bank Angles and Angles of Attack. NASA CR-145004, 1976 